\title{
Face Recognition and Retrieval Using LLBP and URFB
}

\author{
G.Komala Yadav ${ }^{1}$, M.Venkata Ramana ${ }^{2}$ \\ ${ }^{I}$ (Ece,Mrcet/Jntuh, India) \\ ${ }^{2}$ (Ece,Jntuh/ Jntuh, India)
}

\begin{abstract}
Face recognition is one of the major issues in biometric technology. It identifies and/or verifies a person by using a 2D/3D physical characteristics of the face images. Several techniques have been proposed for solving a major problem in face recognition such as fisher face, elastic bunch graph matching and support vector machine. However there are still many challenge problems in face recognition system such as facial expressions, pose variations occlusion and illumination change. Those variations dramatically degrade the performance of face recognition system. It is essential to build an efficient system for face recognition. We introduce a novel face representation method for face recognition integrated with URFB called Local Line Binary Pattern (LLBP) summarizes the local spatial structure of an image by thresholding the local window with binary weight and introduce the decimal number as a texture presentation. Moreover it consumes less computational cost. The basic idea of LLBP is to first obtain the binary code both along the horizontal and vertical directions separately and its magnitude, which characterizes the change in image intensity such as edges and corners, is then computed along with the unified relevance feedback(URFB) shows the advantage over traditional retrieval mechanisms. To seamlessly combine texture feature based retrieval system, a query concept-dependent fusion strategy is automatically learned.

Experimental results on ORL data base consisting of 400 images show that the proposed framework is widely scalable, and effective for recognition, classification and retrieval.
\end{abstract}

Keywords: Binary Code, LLBP, ORL database, Texture, URFB

\section{Introduction}

As the necessity for higher levels of security rises, technology is bound to swell to fulfill these needs. Any new creation, enterprise, or development should be uncomplicated and acceptable for end users in order to spread worldwide. This strong demand for user-friendly systems which can secure our assets and protect our privacy without losing our identity in a sea of numbers, grabbed the attention and studies of scientists toward what's called biometrics.

Biometrics is the emerging area of bioengineering; it is the automated method of recognizing person based on a physiological or behavioral characteristic. There exist several biometric systems such as signature, finger prints, voice, iris, retina, hand geometry, ear geometry, and face. Among these systems, facial recognition appears to be one of the most universal, collectable, and accessible systems.

Biometric face recognition, otherwise known as Automatic Face Recognition (AFR), is a particularly attractive biometric approach, since it focuses on the same identifier that humans use primarily to distinguish one person from another: their "faces". One of its main goals is the understanding of the complex human visual system

The face recognition problem can be divided into two main stages:

- face verification (or authentication), and

- face identification (or recognition).

The detection stage is the first stage; it includes identifying and locating a face in an image. The recognition stage is the second stage; it includes feature extraction, where important information for discrimination is saved, and the matching, where the recognition result is given with the aid of a face database.

Face recognition methods have been proposed. In the vast literature on the topic there are different classifications of the existing techniques. The following is one possible high-level classification:

- Holistic Methods: The whole face image is used as the raw input to the recognition system. An example is the well-known PCA-based technique introduced by Kirby and Sirovich, followed by Turk and Pentland.

- Local Feature-based Methods: Local features are extracted, such as eyes, nose and mouth. Their locations and local statistics (appearance) are the input to the recognition stage. An example of this method is Elastic Bunch Graph Matching (EBGM).

Although progress in face recognition has been encouraging, the task has also turned out to be a difficult endeavor. In the following sections, we give a brief review on technical advances and analyze technical challenges. 


\subsection{History}

Automated face recognition is a relatively new concept. Developed in the 1960s, the first semiautomated system for face recognition required the administrator to locate features ( such as eyes, ears, nose, and mouth) on the photographs before it calculated distances and ratios to a common reference point, which were then compared to reference data. In the 1970s, Goldstein, Harmon, and Lesk used 21 specific subjective markers such as hair color and lip thickness to automate the recognition. The problem with both of these early solutions was that the measurements and locations were manually computed.

In 1988, Kirby and Sirovich applied principle component analysis, a standard linear algebra technique, to the face recognition problem. This was considered somewhat of a milestone as it showed that less than one hundred values were required to accurately code a suitably aligned and normalized face image.

In 1991, Turk and Pentland discovered that while using the eigenfaces techniques, the residual error could be used to detect faces in images; a discovery that enabled reliable real-time automated face recognition systems. Although the approach was somewhat constrained by the environmental factors, the nonetheless created significant interest in furthering development of automated face recognition technologies. The technology first captured the public's attention from the media reaction to a trial implementation at the January 2001 Super Bowl, which captured surveillance images and compared them to a database of digital mugshots. This demonstration initiated much-needed analysis on how to use the technology to support national needs while being considerate of the public's social and privacy concerns.

Today, face recognition technology is being used to combat passport fraud, support law enforcement ,identify missing children, and minimize benefit/identify fraud.

\subsection{Overview}

As one of the most successful applications of image analysis and understanding, face recognition has recently gained significant attention. Over the last ten years or so, it has become a popular area of research in computer vision and one of the most successful applications of image analysis and understanding.

Some examples of face recognition application areas are:

Security

Government Events Criminal

Immigration/Customs

Casino

Toy

Vehicle
Computer and physical access control

Terrorists screening; Surveillance

Illegal immigrant detection; Passport/ ID Card authentication

Filtering suspicious gamblers /VIPs

Intelligent robotic

Safety alert system based on eyelid movement

The largest face recognition systems in the world with over 75 million photographs that is actively used for visa processing operates in the U.S. Department of State.

In 2006, the performance of the latest face recognition algorithms was evaluated in the Face Recognition Grand Challenge. High-resolution face images, 3-D face scans, and iris images were used in the tests. The results indicated that the new algorithms are 10 times more accurate than the face recognition algorithms of 2002 and 100 times more accurate than those of 1995. Some of the algorithms were able to outperform human participants in recognizing faces and could uniquely identify identical twins.

\subsection{Weaknesses vs. Strengths}

Among the different biometric techniques facial recognition may not be the most reliable and efficient but it has several advantages over the others: it is natural, easy to use and does not require aid from the test subject. Properly designed systems installed in airports, multiplexes, and other public places can detect presence of criminals among the crowd. Other biometrics like fingerprints, iris, and speech recognition cannot perform this kind of mass scanning. However, questions have been raised on the effectiveness of facial recognition software in cases of railway and airport security. Critics of the technology complain that the London Borough of Newham scheme has never recognized a single criminal, despite several criminals in the system's database living in the Borough and the system having been running for several years. "Not once, as far as the police know, has Newham's automatic facial recognition system spotted a live target."

Despite the successes of many systems, many issues remain to be addressed. Among those issues, the following are prominent for most systems: the illumination problem, the pose problem, scale variability, images taken years apart, glasses, moustaches, beards, low quality image acquisition, partially occluded faces etc. Figures below show different images which present some of the problems encountered in face recognition. An additional important problem, on top of the images to be recognized, is how different face recognition systems are compared. 


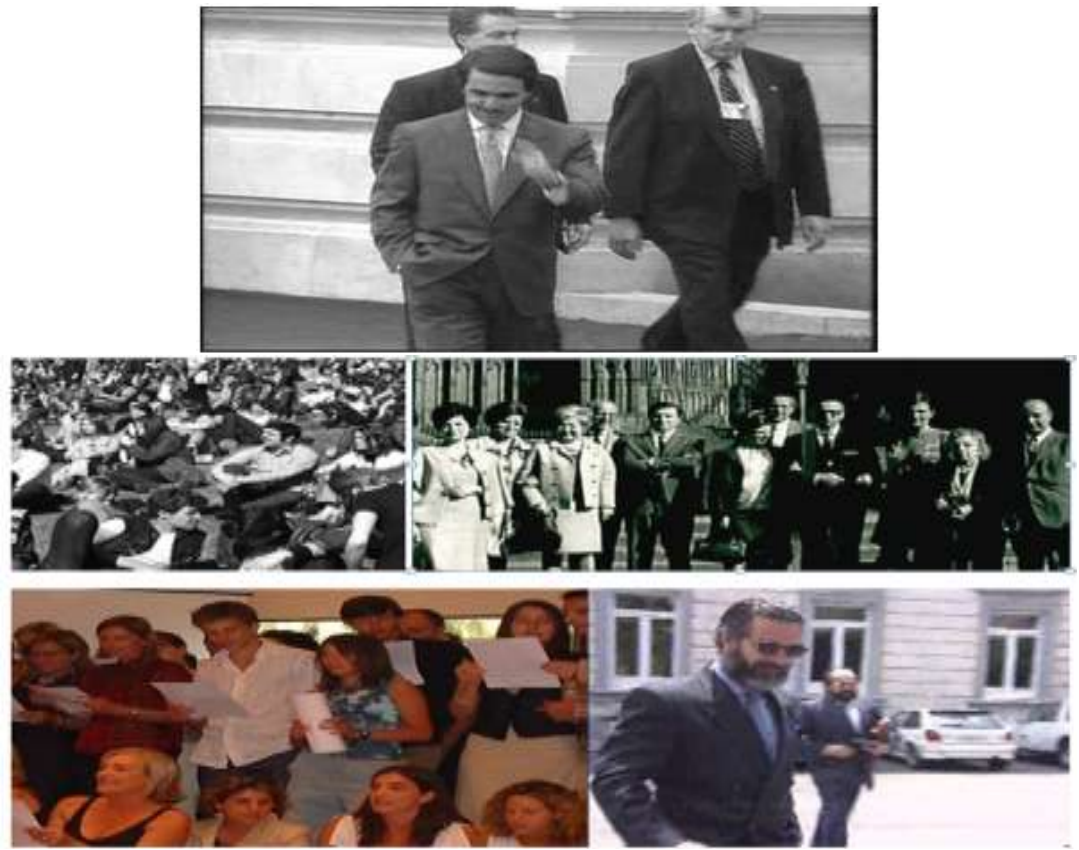

Fig(1):Different images illustrating problems

The illumination problem is illustrated in the following figure, where the same face appears differently due to the change in lighting. More specifically, the changes induced by illumination could be larger than the differences between individuals, causing systems based on comparing images to misclassify the identity of the input image.

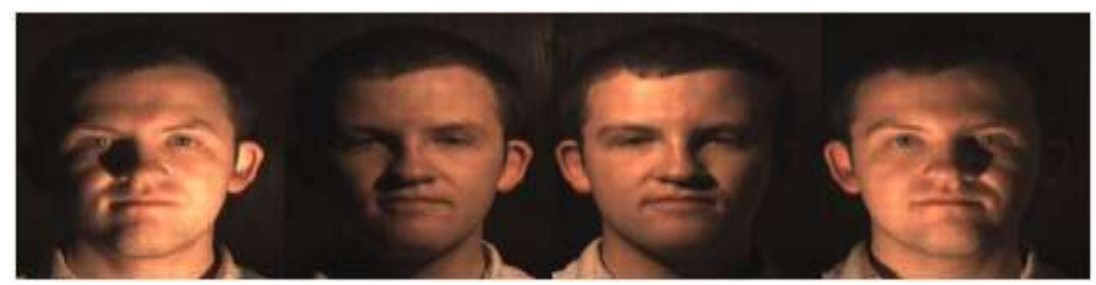

Fig(.2):Illustration of illumination problem

The pose problem is illustrated in the following figure, where the same face appears differently due to changes in viewing condition. The pose problem has been divided into three categories:

- The simple case with small rotation angles,

- The most commonly addressed case, when there is a set of training image pairs (frontal and rotated image

- The most difficult case, when training image pairs are not available and illumination variations are present.

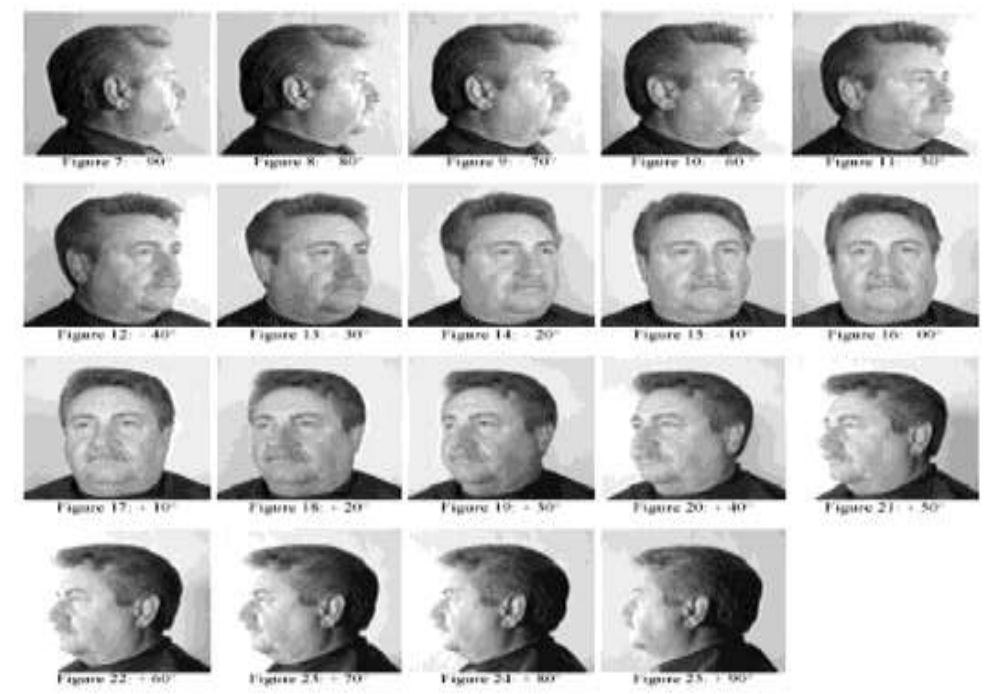

Fig(3): pose problem illustration 
The main aim of this paper includes to recognise a face even with pose variations,illuminations using LLBP and retrieving the 5 relevant images of that particular face and also by giving feedback by the user from the retrieved images results to increase the recognition rate than before feedback.

\section{Binary pattern (image generation)}

Binary pattern is an image produced by a formula that includes binary operations and results in a 32bit integer number. These patterns are closely tied to the $32 \mathrm{bit} \mathrm{RGB}$ color system. These patterns may be used with any integer numbers.

The most simple pattern is $\mathrm{x}$ or $\mathrm{y}$, result of which will be pixel color (in RGB), where $\mathrm{x}$ and $\mathrm{y}$ represent coordinates of image pixel we search.

$\mathrm{x}$ or $\mathrm{y}$ operation where $\mathrm{x}$ and $\mathrm{y}$ are 32bit numbers is per-bit or. For example, if we have 108 (1101100) and 226 (11100010) then 108 or 226 is 238 (11101110). This pattern is not restricted only to $2 \mathrm{D}$, and can be extended into higher dimensions such as $3 \mathrm{D}$.

Uses

- Color generation - possibility to create dozens of new colors, which won't be close to any of basic colors, such as Red, Green, Blue, etc.

- Desktop backgrounds - using several filters its possible to achieve nice patterns, which may be tiled on desktop screen

- Texture generation - different formulas may provide images suitable for procedural image generation, for example creating carpets or walls.

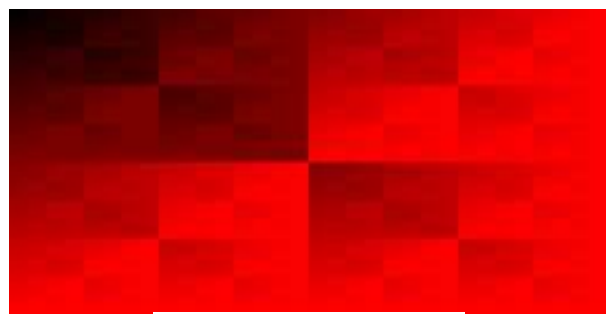

Fig(4):Colour $=\mathrm{x}$ or $\mathrm{y}$

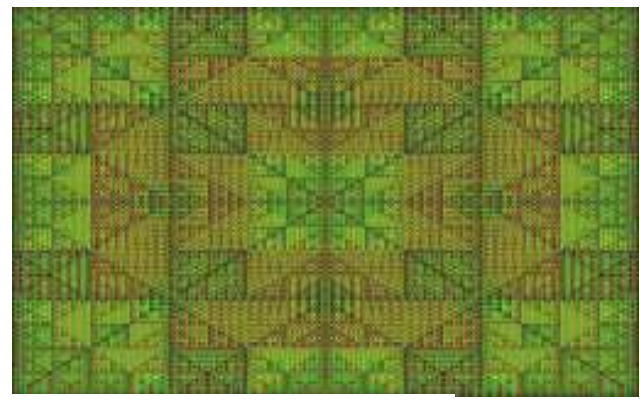

Fig(5):Example of tileable image

\subsection{Local Binary pattern(LBP)}

Local Binary Patterns (LBP) is a type of feature used for classification in computer vision. LBP was first described in 1996. It has since been found to be a powerful feature for texture classification; it has further been determined that when LBP is combined with the Histogram of oriented gradients (HOG) classifier, it yields the best classifier of humans (i.e. person vs. non-person) among the classifiers usually considered in academic literature

The LBP feature vector, in its simplest form, is created in the following manner:

- Divide the examined window to cells (e.g. 16x16 pixels for each cell).

- For each pixel in a cell, compare the pixel to each of its 8 neighbors (on its left-top, left-middle, left-bottom, right-top, etc.). Follow the pixels along a circle, i.e. clockwise or counter-clockwise.

- Where the center pixel's value is greater than the neighbor, write " 1 ". Otherwise, write "0". This gives an 8digit binary number (which is usually converted to decimal for convenience).

- Compute the histogram, over the cell, of the frequency of each "number" occurring (i.e., each combination of which pixels are smaller and which are greater than the center).

- Optionally normalize the histogram.

- Concatenate normalized histograms of all cells. This gives the feature vector for the window. 
The feature vector now can be processed using the Support vector machine or some other machine-learning algorithm, to produce a classifier.

Local binary pattern (LBP) is a popular technique used for image/face representation and classification. LBP has been widely applied in various applications due to its high discriminative power and tolerance against illumination changes such as texture analysis and object recognition. It was originally introduced by Ojala as gray-scale and rotation invariant texture classification. Basically, LBP is invariant to monotonic gray-scale transformations. The basic idea is that each 3x3-neighborhood in an image is threshold by the value of its center pixel and a decimal representation is then obtained by taking the binary sequence (Figure 1) as a binary number such that $L B P \in[0,255]$.

\begin{tabular}{|l|l|l|}
\hline$i_{0}$ & $i_{1}$ & $i_{2}$ \\
\hline$i_{7}$ & $i_{c}$ & $i_{3}$ \\
\hline$i_{6}$ & $i_{5}$ & $i_{4}$ \\
\hline
\end{tabular}

\begin{tabular}{|c|c|c|}
\hline 1 & 2 & 4 \\
\hline 128 & 0 & 8 \\
\hline 64 & 32 & 16 \\
\hline
\end{tabular}

Figure(6): LBP operator: (left) the binary sequence ( 8 bits) and (right) the weighted threshold

\subsection{LOCAL LINE BINARY PATTERN(LLBP)}

The motivation of Local Line Binary Pattern (LLBP) is from Local Binary Pattern (LBP) due to it summarizes the local spacial structure (micro-structure) of an image by thresholding the local window with binary weight and introduce the decimal number as a texture presentation. Moreover it consumes less computational cost.

The basic idea of LLBP is similar to the original LBP but the difference are as follows:

- Its neighbourhood shape is a straight line with length $N$ pixel, unlike in LBP which is a square shape.

- Tthe distribution of binary weight is started from the left and right adjacent pixel of center pixel to the end of left and right side

\subsubsection{System Model}

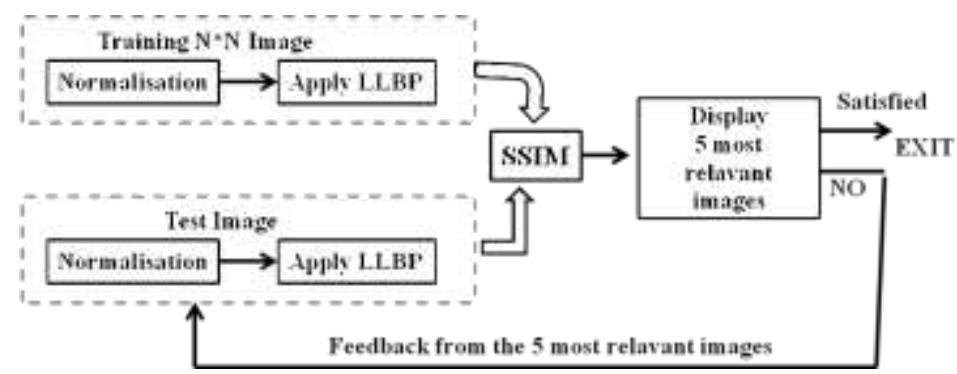

Fig(7): Proposed System model

Both the Test Image and the Training Image are to be compared in order to find the most relevant image from the database. ORL database is choosen where it consists of atmost 4000 images.Before comparing the images (test and training images), Texture content has to be generated for each and every image. These vectors are differnent for different images based on texture properties.

Normalization is the first step to be done while generating the texture content . The given input image is normalized in order to decrease the high contrast intensity values. Each image has to be segmented or to be subdivided in order to apply Local Line Binary Pattern(LLBP).Hence an individual image $\mathrm{N} * \mathrm{~N}$ has been divided into $\mathrm{n} * \mathrm{n}$ (block size).

For each sub block an LLBP operator is applied, since LLBP is an high constrast image. Texture values will differ from image to image.After comparing test and training image by using a quality assement technigue called SSIM(Structural Similarity Index Metric),5 most relevant images were retrieved and displayed.If the result is satisfied by the user then there is no concept of feedback.If the result is nat satisfied by the user,then feedback concept araises.According to user choice,feedback is given from the retrieved images.Feedback may be i.e query or test image may be from the 5 most retrieved images inorder to incresae the search rate or recognition rate. Feedback ia an iterative processes. Till the user satisfied with the result,feedback can be applied to the system.

\subsubsection{Implementation of LLBP algorithm}

The algorithm of LLBP, it first obtains the line binary code along with horizontal and vertical direction seperately and its magnitude, which characterizes the change in image intensity 
such as edges and corners, is then computed, where $L L B P h, L L B P v, L L B P m$ are LLBP on horizontal direction, vertical direction, and its magnitude respectively (The example of processed image for each direction and its magnitude are shown in Figure )
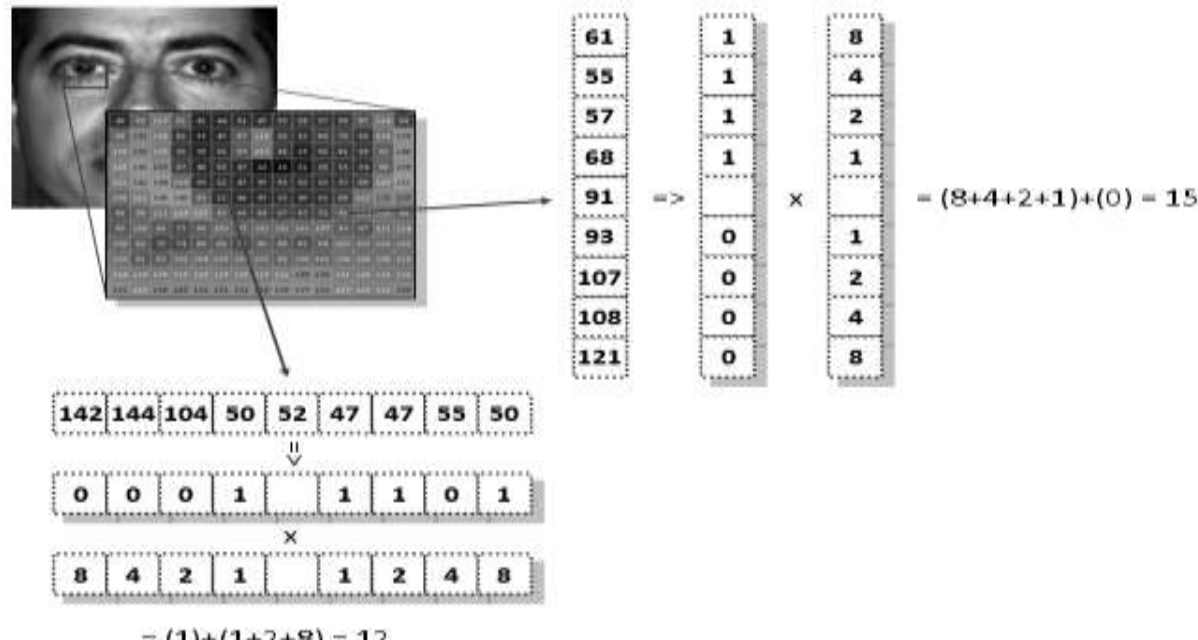

Fig(8): LLBP operator with line length 9 pixels, 8 bits considered

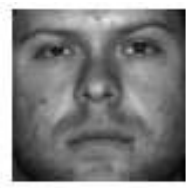

(a)

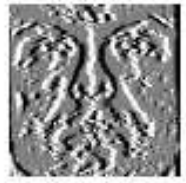

(b)

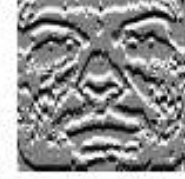

(c)

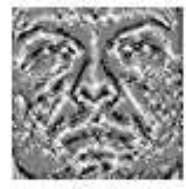

(d)

Fig(9): Example of face image processed by LLBP operator with line length 9 pixels: (a) is original image, (b) and (c) are LLBP along with horizontal and vertical direction, and (d) is its magnitude line in pixel, $c=\left\lceil\frac{N}{2}\right\rceil$ is the position of the center pixel $h c$ on the horizontal line and $v c$ on the vertical line, $h n$ is the pixel along with the horizontal line and $v n$ is the pixel along with the vertical line, and $s$ function is same as in LBP.,

\section{Image retrieval}

An image retrieval system is a computer system for browsing, searching and retrieving images from a large database of digital images. Most traditional and common methods of image retrieval utilize some method of adding metadata such as captioning, keywords, or descriptions to the images so that retrieval can be performed over the annotation words. Manual image annotation is time-consuming, laborious and expensive; to address this, there has been a large amount of research done on automatic image annotation. Additionally, the increase in social web applications and the semantic web have inspired the development of several web-based image annotation tools.

The first microcomputer-based image database retrieval system was developed at MIT, in the 1980s, by Banireddy Prasaad, , Amar Gupta, Hoo-min Toong, and Stuart Madnick.

\subsection{Search methods}

Image search is a specialized data search used to find images. To search for images, a user may provide query terms such as keyword, image file/link, or click on some image, and the system will return images "similar" to the query. The similarity used for search criteria could be meta tags, color distribution in images, region/shape attributes, etc.

- Image meta search - search of images based on associated metadata such as keywords, text, etc.

- Content-based image retrieval (CBIR) - the application of computer vision to the image retrieval. CBIR aims at avoiding the use of textual descriptions and instead retrieves images based on similarities in their contents (textures, colors, shapes etc.) to a user-supplied query image or user-specified image features.

- List of CBIR Engines - list of engines which search for images based image visual content such as color, texture, shape/object, etc. 


\subsection{Data Scope}

It is crucial to understand the scope and nature of image data in order to determine the complexity of image search system design. The design is also largely influenced by factors such as the diversity of user-base and expected user traffic for a search system. Along this dimension, search data can be classified into the following categories:

- Archives - usually contain large volumes of structured or semi-structured homogeneous data pertaining to specific topics.

- Domain-Specific Collection - this is a homogeneous collection providing access to controlled users with very specific objectives. Examples of such a collection are biomedical and satellite image databases.

- Enterprise Collection - a heterogeneous collection of images that is accessible to users within an organization's intranet. Pictures may be stored in many different locations.

- Personal Collection - usually consists of a largely homogeneous collection and is generally small in size, accessible primarily to its owner, and usually stored on a local storage media.

- Web - World Wide Web images are accessible to everyone with an Internet connection. These image collections are semi-structured, non-homogeneous and massive in volume, and are usually stored in large disk arrays.

\subsection{CONTENT-BASED IMAGE RETRIEVAL}

Content-based image retrieval (CBIR), also known as query by image content (QBIC) and contentbased visual information retrieval (CBVIR) is the application of computer vision techniques to the image retrieval problem, that is, the problem of searching for digital images in large databases. (see this survey for a recent scientific overview of the CBIR field) "Content-based" means that the search will analyze the actual contents of the image rather than the metadata such as keywords, tags, and/or descriptions associated with the image. The term 'content' in this context might refer to colors, shapes, textures, or any other information that can be derived from the image itself.

CBIR is desirable because most web based image search engines rely purely on metadata and this produces a lot of garbage in the results. Also having humans manually enter keywords for images in a large database can be inefficient, expensive and may not capture every keyword that describes the image. Thus a system that can filter images based on their content would provide better indexing and return more accurate results. The term Content-Based Image Retrieval (CBIR) seems to have originated in 1992, when it was used by $\mathrm{T}$. Kato to describe experiments into automatic retrieval of images from a database, based on the colors and shapes present. Since then, the term has been used to describe the process of retrieving desired images from a large collection on the basis of syntactical image features. The techniques, tools and algorithms that are used originate from fields such as statistics, pattern recognition, signal processing, and computer vision.

\subsubsection{BACKGROUND \\ Technical progress}

There is a growing interest in CBIR because of the limitations inherent in metadata-based systems, as well as the large range of possible uses for efficient image retrieval. Textual information about images can be easily searched using existing technology, but requires humans to personally describe every image in the database. This is impractical for very large databases, or for images that are generated automatically, e.g. from surveillance cameras. It is also possible to miss images that use different synonyms in their descriptions. Systems based on categorizing images in semantic classes like "cat" as a subclass of "animal" avoid this problem but still face the same scaling issues.

Potential uses for CBIR include:

- Art collections

- Photograph archives

- Retail catalogs

- $\quad$ Medical diagnosis

- Crime prevention

- The military

- Intellectual property

- Architectural and engineering design

- Geographical information and remote sensing systems

CBIR software systems

- University of Washington FIDS Demo

- CIRES: Content Based Image Retrieval System

- LTU-Corbis Visual Search 
- TinEye

- Cortina

- Octagon

- Windsurf

\subsection{CBIR techniques}

Many CBIR systems have been developed, but the problem of retrieving images on the basis of their pixel content remains largely unsolved.

\subsubsection{Query techniques}

Different implementations of CBIR make use of different types of user queries.

a) Query by example

Query by example is a query technique that involves providing the CBIR system with an example image that it will then base its search upon. The underlying search algorithms may vary depending on the application, but result images should all share common elements with the provided example.

Options for providing example images to the system include:

- A preexisting image may be supplied by the user or chosen from a random set.

- The user draws a rough approximation of the image they are looking for, for example with blobs of color or general shapes.

This query technique removes the difficulties that can arise when trying to describe images with words.

b)Other query methods

Other query methods include browsing for example images, navigating customized/hierarchical categories, querying by image region (rather than the entire image), querying by multiple example images, querying by visual sketch, querying by direct specification of image features, and multimodal queries (e.g. combining touch, voice, etc.).

CBIR systems can also make use of relevance feedback, where the user progressively refines the search results by marking images in the results as "relevant", "not relevant", or "neutral" to the search query, then repeating the search with the new information.

\subsection{SEMANTIC RETRIEVAL}

The ideal CBIR system from a user perspective would involve what is referred to as semantic retrieval, where the user makes a request like "find pictures of dogs" or even "find pictures of Abraham Lincoln". This type of open-ended task is very difficult for computers to perform - pictures of chihuahuas and Great Danes look very different, and Lincoln may not always be facing the camera or in the same pose. Current CBIR systems therefore generally make use of lower-level features like texture, color, and shape, although some systems take advantage of very common higher-level features like faces (see facial recognition system). Not every CBIR system is generic. Some systems are designed for a specific domain, e.g. shape matching can be used for finding parts inside a CAD-CAM database.

\subsection{Content comparison using image distance measures}

The most common method for comparing two images in content based image retrieval (typically an example image and an image from the database) is using an image distance measure. An image distance measure compares the similarity of two images in various dimensions such as color, texture, shape, and others.

For example a distance of 0 signifies an exact match with the query, with respect to the dimensions that were considered. As one may intuitively gather, a value greater than 0 indicates various degrees of similarities between the images. Search results then can be sorted based on their distance to the queried image.

\section{a) COLOR}

Computing distance measures based on color similarity is achieved by computing a color histogram for each image that identifies the proportion of pixels within an image holding specific values (that humans express as colors). Current research is attempting to segment color proportion by region and by spatial relationship among several color regions. Examining images based on the colors they contain is one of the most widely used techniques because it does not depend on image size or orientation. Color searches will usually involve comparing color histograms, though this is not the only technique in practice.

\section{b) TEXTURE}

Texture measures look for visual patterns in images and how they are spatially defined. Textures are represented by texels which are then placed into a number of sets, depending on how many textures are detected in the image. These sets not only define the texture, but also where in the image the texture is located. 
Texture is a difficult concept to represent. The identification of specific textures in an image is achieved primarily by modeling texture as a two-dimensional gray level variation. The relative brightness of pairs of pixels is computed such that degree of contrast, regularity, coarseness and directionality may be estimated (Tamura, Mori \& Yamawaki, 1978). However, the problem is in identifying patterns of co-pixel variation and associating them with particular classes of textures such as silky, or rough.

\section{c) SHAPE}

Shape does not refer to the shape of an image but to the shape of a particular region that is being sought out. Shapes will often be determined first applying segmentation or edge detection to an image.

Other methods like [Tushabe and Wilkinson 2008] use shape filters to identify given shapes of an image. In some case accurate shape detection will require human intervention because methods like segmentation are very difficult to completely automate.

\subsection{Applications}

Some software producers are trying to push CBIR based applications into the filtering and law enforcement markets for the purpose of identifying and censoring images with skin-tones and shapes that could indicate the presence of nudity, with controversial results.

\subsection{What is texture?}

Everyday texture terms - rough, silky, bumpy - refer to touch.

A texture that is rough to touch has:

- a large difference between high and low points, and

- a space between highs and lows approximately the same size as a finger.

Silky would have

- little difference between high and low points, and

- the differences would be spaced very close together relative to finger size.

Image texture works in the same way, except the highs and lows are brightness values (also called grey levels, GL, or digital numbers, DN) instead of elevation changes. instead of probing a finger over the surface, a "window" - a (usually square) box defining the size of the probe - is used.

\subsubsection{Textures in images quantify:}

- $\quad$ Grey level differences (contrast)

- Defined size of area where change occurs (window)

- Directionality or lack of it

\subsubsection{GLCM}

Grey-Level Co-occurrence Matrix texture measurements have been the workhorse of image texture since they were proposed by Haralick in the 1970s. To many image analysts, they are a button you push in the software that yields a band whose use improves classification - or not. The original works are necessarily condensed and mathematical, making the process difficult to understand for the student or front-line image analyst. This GLCM texture tutorial was developed to help such people, and it has been used extensively worldwide since 1999.

This document concerns some of the most commonly used texture measures, those derived from the Grey Level Co-occurrence Matrix (GLCM). The essence is understanding the calculations and how to do them. This involves

- Defining a Grey Level Co-occurrence Matrix (GLCM)

- Creating a GLCM

- Using it to calculate texture in the exercises.

- Understanding how calculations are used to build up a texture image

- Viewing examples of the texture images created with various input parameters

\section{Definition: Order:}

The GLCM described here is used for a series of "second order" texture calculations.

First order texture measures are statistics calculated from the original image values, like variance, and do not consider pixel neighbor relationships.

Second order measures consider the relationship between groups of two (usually neighboring) pixels in the original image. 
Third and higher order textures (considering the relationships among three or more pixels) are theoretically possible but not commonly implemented due to calculation time and interpretation difficulty. There has been some recent development of a more efficient way to calculate third-order textures.

\subsubsection{Framework for the GLCM:}

\section{Spatial relationship between two pixels:}

GLCM texture considers the relation between two pixels at a time, called the reference and the neighbour pixel. In the illustration below, the neighbour pixel is chosen to be the one to the east (right) of each reference pixel. This can also be expressed as a $(1,0)$ relation: 1 pixel in the $\mathrm{x}$ direction, 0 pixels in the $\mathrm{y}$ direction.

Each pixel within the window becomes the reference pixel in turn, starting in the upper left corner and proceeding to the lower right. Pixels along the right edge have no right hand neighbour, so they are not used for this count.

\subsubsection{Properties of the GLCM}

1.It is square:

The reference pixels have the same range of values as the neighbour pixels, so the values along the top are identical to the values along the side.

2.Has the same number of rows and columns as the quantization level of the image:

The test image has four grey level values $(0,1,2$ and 3$)$. Eight bit data has 256 possible values, so would yield a 256 × 256 square matrix, with 65,536 cells. 16 bit data would give a matrix of size 65536 x $65536=$ $429,496,720$ cells!

3. It is symmetrical around the diagonal:

Some things to notice about the normalized symmetrical GLCM (called the GLCM from here on)

The diagonal elements all represent pixel pairs with no grey level difference (0-0, 1-1, 2-2, 3-3 etc.). If there are high probabilities in these elements, then the image does not show much contrast: most pixels are identical to their neighbours.

When values in the diagonal are summed, the result is the probability of any pixel's being the same grey level as its neighbour.

Look at lines parallel to the diagonal. Cells one cell away from the diagonal represent pixel pairs with a difference of only one grey level (0-1, 1-2, 2-3 etc.). Similarly, values in cells two away from the diagonal show how many pixels have 2 grey level differences, and so forth. The farther away from the diagonal, the greater the difference between pixel grey levels.

Sum up these parallel diagonals and the result is the probability of any pixel's being 1 or 2 or 3 etc. different from its neighbour.

\subsubsection{Calculating texture measures from the GLCM}

Most texture calculations are weighted averages of the normalized GLCM cell contents.

A weighted average multiplies each value to be used by a factor (a weight) before summing and dividing by the number of values. The weight is intended to express the relative importance of the value.

Example: the most common weighted average that students encounter is the term grade. Exams usually have a higher weight than quizzes. The weights are the $\%$ of course grade assigned to each mark.

\subsubsection{Creating a texture image}

The result of a texture calculation is a single number representing the entire window. This number is put in the place of the centre pixel of the window, then the window is moved one pixel and the process is repeated of calculating a new GLCM and a new texture measure. In this way an entire image is built up of texture values.

Edge of image problems Each cell in a window must sit over an occupied image cell. This means that the centre pixel of the window cannot be an edge pixel of the image. If a window has dimension $\mathrm{N} \mathrm{x} \mathrm{N}$, a strip $(\mathrm{N}-1) / 2$ pixels wide around the image will remain unoccupied. The usual way of handling this is to fill in these edge pixels with the nearest texture calculation. 


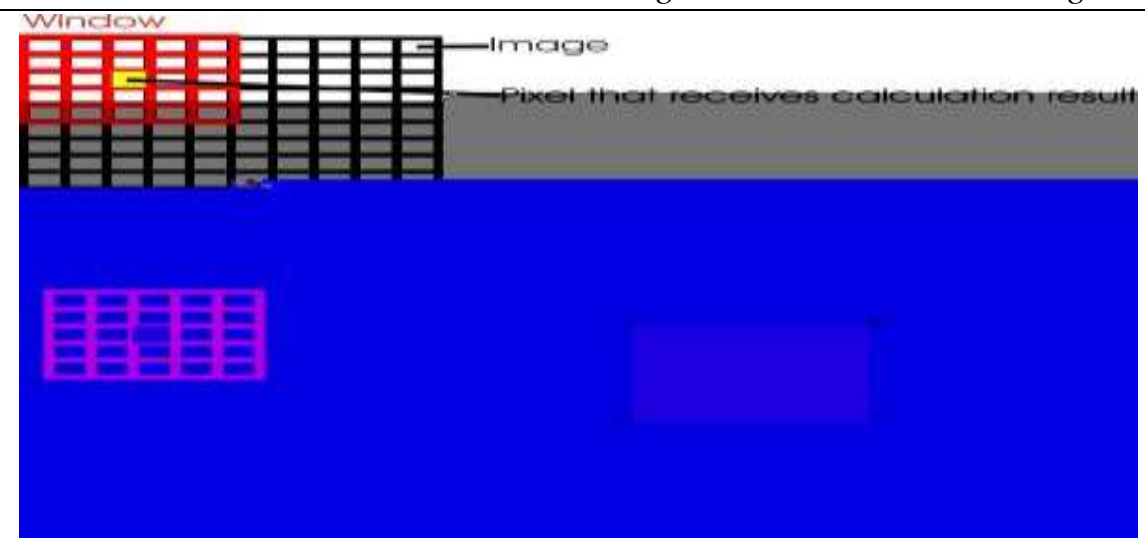

Fig(10):Creating a texture image

Image edge pixels usually represent a very small fraction of total image pixels, so this is only a minor problem. However, if the image is very small or the window is very large, the image edge effect should be remembered Edge effects can be a problem in classification.

\subsection{RF in textual space}

CBIR systems perform retrieval based on the similarity defined in terms of visual features with more objectiveness. Although some new methods, such as the relevant feedback, have been developed to improve the performance of CBIR systems, low-level features do still play an important role and in some sense be the bottleneck for the development and application of CBIR techniques.

A very basic issue in designing a CBIR system is to select the most effective image features to represent image contents. Many low-level features have been researched so far. Currently, the widely used features include color features, such as color correlogram, color moments, color histogram, and texture features, such as Gabor wavelet feature, MR-SAR. As the color and texture features capture different aspects of images, their combination may be useful.

However, despite many research efforts, the existing low-level features are still not powerful enough to represent image content. Some features can achieve relatively good performance, but their feature dimensions are usually too high, or the implementation of the algorithm is difficult.

\subsection{APPLICATIONS}

The CBIR technology has been used in several applications such as fingerprint identification, biodiversity information systems, digital libraries, crime prevention, medicine, historical research, among others. Some of these applications are presented in this section.

\section{Medical Applications}

The use of CBIR can result in powerful services that can benefit biomedical information systems. Three large domains can instantly take advantage of CBIR techniques: teaching, research, and diagnostics [73]. From the teaching perspective, searching tools can be used to find important cases to present to students. Research also can be enhanced by using services combining image content information with different kinds of data.

For example, scientists can use mining tools to discover unusual patterns among textual (e.g., treatments reports, and patient records) and image content information. Similarity queries based on image content descriptors can also help the diagnostic process. Clinicians usually use similar cases for case-based reasoning in their clinical decision-making process.

In this sense, while textual data can be used to find images of interest, visual features can be used to retrieve relevant

\section{Biodiversity Information Systems}

Biologists gather many kinds of data for biodiversity studies, including spatial data, and images of living beings. Ideally, Biodiversity Information Systems (BIS) should help researchers to enhance or complete their knowledge and understanding about species and their habitats by combining textual, image content-based, and geographical queries.

An example of such a query might start by providing an image as input (e.g., a photo of a fish) and then asking the system to "Retrieve all database images containing fish whose fins are shaped like those of the fish in this photo". 
A combination of this query with textual and spatial predicates would consist of "Show the drainages where the fish species with 'large eyes' coexists with fish whose fins are shaped like those of the fish in the photo". Examples of initiatives in this area include [55, 74].

\section{Digital Libraries}

There are several digital libraries that support services based on image content [74-79]. One example is the digital museum of butterflies [74], aimed at building a digital collection of Taiwanese butterflies. This digital library includes a module responsible for content-based image retrieval based on color, texture, and patterns. In a different image context, Zhu et al. [76] present a content-based image retrieval digital library that supports geographical image retrieval. The system manages air photos which can be retrieved through texture descriptors. Place names associated with retrieved images can be displayed by cross referencing with a Geographical Name Information System (GNIS) gazetter.

In this same domain, Bergman et al. describe an architecture for storage and retrieval of satellite images and video data from a collection of heterogeneous archives. Other initiatives cover different concepts of the CBIR area. For example, while research presented in [77,78] concentrates on new searching strategies for improving the effectiveness of CBIR systems, another popular focus is on proposing image descriptors [79].

\section{Relevance Feedback}

Search systems operate using a standard retrieval model, where a searcher, with a need for information, searches for documents that will help supply this information. Searchers are typically expected to describe the information they require via a set of query words submitted to the search system. This query is compared to each document in the collection, and a set of potentially relevant documents is returned. It is rare that searchers will retrieve the information they seek in response to their initial retrieval formulation (Van Rijsbergen, 1986). However, such problems can be resolved by iterative, interactive techniques. The initial query can be reformulated during each iteration either explicitly by the searcher or based on searcher interaction.

The direct involvement of the searcher in interactive IR results in a dialogue between the IR system and the searcher that is potentially muddled and misdirected (Ingwersen, 1992). Searchers may lack a sufficiently developed idea of what information they seek and may be unable to conceptualize their needs into a query statement understandable by the search system. When unfamiliar with the collection of documents being searched they may have insufficient search experience to adapt their query formulation strategy (Taylor, 1968; Kuhlthau, 1988), and it is often necessary for searchers to interact with the retrieval system to clarify their query.

Relevance feedback (RF) is a technique that helps searchers improve the quality of their query statements and has been shown to be effective in non-interactive experimental environments (e.g., Salton and Buckley, 1990) and to a limited extent in IIR (Beaulieu, 1997). It allows searchers to mark documents as relevant to their needs and present this information to the IR system. The information can then be used to retrieve more documents like the relevant documents and rank documents similar to the relevant ones before other documents (Ruthven, 2001, p. 38). RF is a cyclical process: a set of documents retrieved in response to an initial query are presented to the searcher, who indicates which documents are relevant.

This information is used by the system to produce a modified query which is used to retrieve a new set of documents that are presented to the searcher. This process is known as an iteration of RF, and repeats until the required set of documents is found.

To work effectively, RF algorithms must obtain feedback from searchers about the relevance of the retrieved search results. This feedback typically involves the explicit marking of documents as relevant. The system takes terms from the documents marked and these are used to expand the query or re-weight the existing query terms. This process is referred to as query modification. The process increases the score of terms that occur in relevant documents and decreases the weights of those in non relevant documents. The terms chosen by the RF system are typically those that discriminate most between the documents marked and those that are not. The query statement that evolves can be thought of as a representation of a searcher's interests within a search session (Ruthven et al., 2002a).

The classic model of IR involves the retrieval of documents in response to a query devised and submitted by the searcher. The query is a one-time static conception of the problem, where the need assumed constant for the entire search session, regardless of the information viewed. RF is an iterative process to improve a search system's representation of a static information need. That is, the need after a number of iterations is assumed to be the same as at the beginning of the search (Bates, 1989). The aim of RF is not to provide information that enables a change in the topic of the search.

The evolution of the query statement across a number of feedback iterations is best viewed as a linear process, resulting in the formulation of an improved query. Initially, this model of RF was not regarded as an interaction between searcher and system and a potential source of relevance information. However current accounts of 
feedback in IIR expand the notion of feedback to one in which the system and the searcher engage in direct dialogue, with feedback flowing from searcher to system and vice-versa (Spink and Losee, 1996).

The value of IIR systems that use RF over systems that do not offer RF has already been established (Koenemann and Belkin, 1996). As this study demonstrates, it is possible to gain a deeper understanding of what searchers want from RF systems through empirical investigation.

A number of studies have found that searchers exhibit a desire for explicit relevance feedback features and, in particular, term suggestion features (Hancock-Beaulieu and Walker, 1992; Koenemann and Belkin, 1996; Beaulieu, 1997; Belkin et al., 2000). However, evidence from these and related studies have indicated that the features of RF systems are not used in interactive searching (Beaulieu, 1997; Belkin et al., 2001; Ruthven et al., 2001); there appears to be an inconsistency between what searchers say they want and what they actually use when confronted with RF systems. Searchers may lack the cognitive resources to effectively manage the additional requirements of the marking documents whilst trying to complete their search task. The interface support for explicit RF can often take the form of checkboxes next to each document at the interface, allowing searchers to mark documents as relevant, or a sliding scale that allows them to indicate the extent to which a document is relevant (Ruthven et al., 2002b). The process of indicating which information is relevant is unfamiliar to searchers, and is adjunct to the activity of locating relevant information. The feedback mechanism is not implemented as part of the routine search activity; searchers may forget to use the feature or find it too onerous (Furnas, 2002).

Despite the apparent advantages of RF there have been relatively few attempts to implement it in a full commercial environment. Aalbersberg (1992) cited two possible reasons for this trend; the high computational load necessitated by the RF algorithms and unfriendliness of the RF interface. With recent improvements in processing power, the computational expense is no longer of real concern. Although the ser interface challenge remains, technological advances mean that interfaces can be onstructed that make RF more easily understood by searchers (Tague and Schultz, 1988; Gauch, 1992)

In operational environments searchers may be unable or unwilling to visit documents to assess their relevance. Documents may be lengthy or complex, searchers may have time restrictions or the initial query may have retrieved a poor set of documents. In RF systems the searcher is only able to judge the relevance of the documents that are presented to them. If a small number of relevant documents are retrieved then the ability of the system to approximate the searcher's information need (via modified queries taken from searchers' relevance judgements) can be adversely affected.

Relevance is an 'intuitive' concept (Saracevic, 1996) of which there are many different types (Mizzaro, 1998), and as such is not easy to define or measure. Traditional RF systems use a binary notion of relevance: either a document is relevant, or it is not. This is an overly simplified view of what is an implicitly variable and immeasurable concept. Many studies in IR have either used binary notions of relevance directly (Rees, 1967; Schamber et al., 1990), or collapsed more complex scales (incorporating the 'fuzzy regions of relevance' (Spink et al., 1998)) into binary scales for analysis purposes (Saracevic et al., 1988; Schamber, 1991; Pao, 1993).

Partial relevance, despite its usefulness (Spink et al., 1998) is typically ignored in RF systems since the formulae used to select query expansion terms and re-weight existing terms use a binary notion of relevance. There is therefore a need to incorporate less concrete, more fuzzy notions of relevance into the term selection process that underlies RF (Ruthven et al., 2002b).

Another potential application of RF techniques is in negative relevance feedback; the selection of important terms in non-relevant documents that are then de-emphasized or removed completely from the query. This approach has been shown to not detract from, and may improve, searching behavior when used in interactive IR applications (Belkin et al., 1996a; 1998). In these studies it was suggested that the technique was difficult to use, not helpful and its effectiveness was dependent on the search topic. This may be due to how negative relevance feedback was supported at the interface.

The RF features investigated in some of the studies described in this section may have been influenced by the environment in which they were evaluated (i.e., in a controlled, laboratory setting). In a study looking at different types of query expansion techniques, Dennis et al. (1998) found that although searchers could successfully use novel expansion techniques and could be convinced of the benefits of these techniques in a laboratory or training environment, they often stopped using these techniques in operational environments. Anick (2003) recently found in a Web-based study, that many searchers made use of a term suggestion feature to refine their query. The results suggest the potential of term suggestion features, in some types of searching environments, especially for single session interactions. The different findings in these two studies suggest that RF may be situation-dependent and that many factors other than its usefulness influence its use. In the next section techniques to help searchers use RF systems are discussed.

Relevance feedback, originally developed for information retrieval, is an online learning technique aiming at improving the effectiveness of the information retrieval system. The main idea of relevance feedback is to let the user guide the system. 
During retrieval process, the user interacts with the system and rates the relevance of the retrieved documents, according to his/her subjective judgment. With this additional information, the system dynamically learns the user's intention, and gradually presents better results. Since the introduction of relevance feedback to image retrieval in the mid-1990s, it has attracted tremendous attention in the content-based image retrieval (CBIR) community and has been shown to provide dramatic performance improvement. However, no commercial Web image search engines support relevance feedback because of usability, scalability, and efficiency issues.

\subsection{Implicit feedback}

Implicit feedback systems make inferences of what is relevant based on searcher interaction and do not intrude on the searcher's primary line of activity i.e., satisfying their information needs (Furnas, 2002). In traditional relevance feedback systems, the function of making judgments is intentional, and specifically for the purpose of helping the system build up a richer body of evidence on what information is relevant. However, the ultimate goal of information seeking is to satisfy an information need, not to rate documents. Systems that use implicit feedback to model information needs and enhance search queries fit better with this goal.

Implicit feedback systems typically use measures such as document reading time, scrolling and interaction to make decisions on what information is relevant (Claypool et al., 2001). However, these systems typically assume that searchers will view and interact with relevant documents more than non-relevant documents. These assumptions are context-dependent and vary greatly between searchers. The approach used for implicit feedback makes a potentially more robust assumption: searchers will try to view relevant information. Through monitoring the information searchers interact with search systems can approximate their interests. This is made possible since the interface components the search interfaces present are smaller than the full-text of documents, allowing relevance information to be communicated more accurately.

In TRS Feedback and TRS Document some of the experimental systems use evidence gathered via implicit feedback to restructure the retrieved information during the search. In these systems, each retrieved document has an associated summary composed of the best four Top- Ranking Sentences that appear on the interface at the searcher's request. The viewing of this summary is regarded as an indication of interest in the information it contains and is used as an indication of relevance.

These relevance indications are used by the systems to reorder the Top-Ranking Sentences. Sentences are small and the differences in sentence scores between sentences are also small. Should there be a slight change in the system's formulation of the information need a list of sentences is much more likely to change than, say, a list of documents. At no point, in any experimental system, is the searcher shown the expanded query; they are only shown the effect of the query (i.e., the reordered top-ranking sentence list).

Reordering the sentence list based on implicit feedback means it represents the system's current estimation of the searcher's interests. Since this formulation is based solely on the viewed information the system is able to form reasonable approximations on what information is relevant. As the searcher becomes more sure of their need, or indeed as the need changes, the search system can adapt, select new query terms and use this query to update the ordering of the Top- Ranking Sentences list to reflect this change.

The traditional view of information seeking assumes a searcher's need is static and represented by a single query submitted at the start of the search session. However, as is suggested by Harter (1992) among others, the need is in fact dynamic and changes to reflect the information viewed during a search. As they view this content their knowledge changes and so does their problematic situation. It is therefore preferable to express this modified problem with a revised query. The experimental systems in TRS Feedback and TRS Document do this, selecting the most useful query expansion terms during a search.

In the systems developed in these studies, the sentences are reordered using implicit relevance information gathered unobtrusively from searcher interaction. Experimental subjects found this a useful feature that helped them find relevant information. They suggested that it was most useful when they felt the initial query had retrieved a large amount of potentially relevant information and they wanted to focus their attention on only the most relevant parts. These are more push oriented than the static Top-Ranking Sentences system tested in TRS Presentation. The systems are adaptive, work to better represent information needs and consider changes in these needs, restructuring the content presented at the results interface.

In TRS Feedback and TRS Document assumed that the viewing of a document's summary was an indication of an interest in the relevance of the summary's contents. There are several grounds on which this can be criticized; searchers will view non-relevant summaries, the title rather than the summary was what the user expressed an interest in, and the searcher may look at all retrieved documents before making real relevance decisions. Nevertheless I felt that this assumption was fair enough to allow an initial investigation into the use of implicit feedback. In TRS Document I introduced a timing mechanism to eliminate the problems caused by the accidental 'mouse over' of document titles and the unwanted removal of sentences from the Top-Ranking Sentences list that follows. The results of TRS Document are testament to the success of a very limited version of an implicit feedback technique. More complex and effective techniques based on these findings are described in later chapters of this thesis. 
Despite their positive comments, subjects had two reservations about how system decisions based on implicit feedback were communicated. Firstly, since the reordering occurred at the same time as a summary appeared or updated they did not always notice the effect of the reordering. The presentation of the updating therefore needs improving in future systems. Secondly, the Top-Ranking Sentences only contained sentences from Web pages for which the subject had not already viewed a summary. If the subject viewed the summary for a page, then all sentences from that page were removed from the list of Top-Ranking Sentences. This choice was made to increase the degree to which the list of Top-Ranking Sentences would update.

However, many subjects stated that they would prefer less updating and no removal of sentences. In White (2004) I proposed the use of Scroll Tiles to communicate the effects of the sentence reordering using a familiar interface component, the scrollbar. The approach represented sentences as tiles on the scrollbar and recolored the tiles to represent changes in the ordering. A pilot study was conducted that involved nine experimental subjects and compared systems that re-colored a representation of the sentences imposed on the scrollbar with one that reordered the actual sentences. The Scroll Tiles were shown to be more effective for conveying reordering decisions than the sentence updating. However, they are not used in any further interfaces described in this thesis as I tried to limit the number of new interface components to only those necessary to test experimental hypotheses.

The results of the three studies show that it is possible to get searchers to interact with more than a few search results. The approach moves away from simply presenting titles to presenting alternative access methods for assessing and targeting potentially relevant information.

RF systems suffer from a number of problems that make effective alternatives appealing. Implicit feedback techniques unobtrusively infer information needs based on search behavior, and can be used to individuate system responses and build models of system users. Implicit feedback techniques have been used to retrieve, filter and recommend different types of document (e.g., Web documents, email messages, newsgroup articles) from a variety of online sources. The research described in this section is limited to the use of implicit feedback techniques for information retrieval related tasks.

Some of the surrogate measures (or behaviors) that have been most extensively investigated as sources of implicit feedback include reading time, saving, printing, selecting and referencing (Morita and Shinoda, 1994; Konstan et al., 1997; Joachims et al., 1997; Billsus and Pazzani, 1999; Seo and Yang, 2000). The primary advantage in using implicit techniques is that they remove the cost to the searcher of providing feedback. Implicit measures are generally thought to be less accurate than explicit measures (Nichols, 1997) but as described in the previous section if implemented carefully can be effective substitutes for them (White et al., 2002b). Since large quantities of implicit data can be gathered at no extra cost to the searcher, they are attractive alternatives to explicit techniques. Moreover,implicit measures can be combined with explicit ratings to obtain a more accurate representation of searcher interests.

In general, the application of implicit measures does not consider the characteristics of individual searchers. All searchers are assumed to exhibit stereotypical search behaviors around relevant information. One of the most widely used behaviours for implicit modeling is reading time (Morita and Shinoda, 1994; Konstan et al., 1997; Billsus and Pazzani, 1999; Seo and Yang, 2000; White et al., 2002a). This has been questioned for being too simplistic and not taking full account the influencing effects of other factors such as task, topic and user characteristics (Kelly and Belkin, 2001; 2002).

In a related study Kelly and Cool (2002) found that as topic familiarity increased, reading time decreased, and proposed that as the searcher's state of knowledge increased, their search behavior altered. Such findings suggest a role for different relevance indications at different points in the search session, based on topic familiarity. Kelly (2004) suggested that to develop models of document preference, techniques based on implicit feedback must also be able models the searcher's information seeking context and must construct models that are personal to the searcher, not general, for all searchers. Kelly also found in the same naturalistic user study that despite its popularity as an implicit feedback measure document retention is not a good indicator of document preference.

Searchers may retain a document for a number of reasons, only one of which is the relevance of its content. Morita and Shinoda (1994) conducted a longitudinal study of search behaviors when reading newsgroup documents. Over a period of time, subjects were required to view newsgroup documents and explicitly rate their interest in the articles. The authors examined reading time and keeping behaviors of experimental subjects.

Researchers have found a positive relationship between reading time and user interests, but none between retention and document interests. In a related study Goecks and Shavlik (2000) measured hyperlinks clicked, scrolling performed and processor cycles used to unobtrusively predict the interests of a searcher. They integrated these measures into an agent that employed a neural network and showed that it could predict user activity and build a model of their interests that could be used to search the Web on their behalf.

There exits a novel low-level feature, named color texture moments, for representing image contents. It is able to integrate the color and texture characteristics of an image in one compact form. Preliminary experimental 
results show that the new feature achieves better performance than many existing low-level features. More importantly, the dimension of this new feature is only 48, much lower than that of many features with good performance. Furthermore, the feature extraction algorithm is very easy to implement. It is valuable for the development and application of the CBIR systems.

A texture feature based on the local Fourier transform (LFT) has been developed to classify textures and segment images. We operate the original image with eight templates derived from LFT and obtain eight characteristic maps, each of which characterizes some information on a certain aspect of the original image. Similar to color moments, we calculate the first and second moments of the characteristic maps, which represent the color texture information of the original

\section{2. $R F$ in textual space}

Rocchio's algorithm is used. The algorithm was developed in the mid-1960s and has been proven to be one of the most effective RF algorithms in information retrieval. The key idea of Rocchio's algorithm is to construct a so-called optimal query so that the difference between the average score of a relevant document and the average score of a non relevant document is maximized. Cosine similarity is used to calculate the similarity between an image and the optimal query. Since only clicked images are available for our proposed framework,

\subsection{RF in visual space:}

To perform RF in visual space, Rui's algorithm is used. Assume clicked images to be relevant, both an optimal query and feature weights are learned from the clicked images. More specifically, the feature vector of the optimal query is the mean of all features of clicked images. The weight of a feature dimension is proportional to the inverse of the standard deviation of the feature values of all clicked images. Weighted Euclidean distance is used to calculate the distance between an image and the optimal query. Although Rui's algorithm is used currently, any RF algorithm using only relevant images could be used in the unified framework.

Relevance feedback (RF) has been extensively studied in the content-based image retrieval community. However, no commercial Web image search engines support RF because of scalability, efficiency and effectiveness issues. In this paper we proposed a scalable relevance feedback mechanism using click through data for web image retrieval. The proposed mechanism regards users' click-through data as implicit feedback which could be collected at lower cost, in larger quantities and without extra burden on the user.

During RF process, both textual feature and visual feature are used in a sequential way. To seamlessly combine textual feature-based RF and visual feature-based RF, a query concept-dependent fusion strategy is automatically learned. Experimental results on a database consisting of nearly three million Web images show that the proposed mechanism is wieldy, scalable and effective.

With the explosive growth of both World Wide Web and the number of digital images, there is more and more urgent need for effective Web image retrieval systems.

Most of the popular commercial search engines, such as Google, Yahoo! And AltaVista, support image retrieval by keywords.

There are also commercial search engines dedicated to image retrieval, e.g. Pic search. A common limitation of most of the existing Web image retrieval systems is that their search process is passive, i.e disregarding the informative interactions between users and retrieval systems.

An active system should let the user involve into the loop so that personalized results could be provided for specific users. To be active, the system could use relevance feedback techniques.

Relevance feedback, originally developed for information retrieval is an online learning technique used to improve the effectiveness of the information retrieval system. The main idea of relevance feedback is to let the user guide the system. During retrieval process, the user interacts with the system and rates the relevance of the retrieved documents, according to his/her subjective judgment. With this additional information, the system dynamically learns the user's intention, and gradually presents better results.

Since the introduction of relevance feedback into image retrieval in the mid-1990, it has attracted tremendous attention in the CBIR community and has been shown to provide dramatic performance improvement. However, almost all the existing relevance feedback algorithms in image retrieval systems are performed in an explicit way. It is noted that explicit relevance feedback techniques have been underutilized as they place an increased cognitive burden on users while the benefits are not always obvious to them. Comparing with explicit feedback, implicit feedback could be collected at much lower cost, in much larger quantities and without burden on the user. As one of the most effective implicit feedback information, click-through data has been used either as absolute relevance judgments or relative relevance judgments.

To the best of our knowledge, implicit feedback using click through data has not been applied to Web image retrieval systems in both textual space and visual space. Comparing with text retrieval, image retrieval has the following two characteristics. First, since the thumbnail of an image reflects more information than the 
title and snippet of a Web page, click-through information of image retrieval tends to be less noisy than that of text retrieval.

Second, unlike textual document, the content of an image can be taken in at a glance. As a result, the user will possibly click more results in image retrieval than in page retrieval. Both characteristics imply that click-through data could be more helpful for image retrieval. Thus there has been study on generating semantic features ba

The approach used for implicit feedback makes a potentially more robust assumption: searchers will try to view relevant information. Through monitoring the information searchers interact with search systems can approximate their interests. This is made possible since the interface components the search interfaces present are smaller than the full-text of documents, allowing relevance information to be communicated more accurately.

Relevance feedback, originally developed for information retrieval is an online learning technique used to improve the effectiveness of the information retrieval system. The main idea of relevance feedback is to let the user guide the system. During retrieval process, the user interacts with the system and rates the relevance of the retrieved documents, according to his/her subjective judgment. With this additional information, the system dynamically learns the user's intention, and gradually presents better results.

Since the introduction of relevance feedback into image retrieval in the mid-1990, it has attracted tremendous attention in the CBIR community and has been shown to provide dramatic performance improvement.

However, almost all the existing relevance feedback algorithms in image retrieval systems are performed in an explicit way. It is noted that explicit relevance feedback techniques have been underutilized as they place an increased cognitive burden on users while the benefits are not always obvious to them. Comparing with explicit feedback, implicit feedback could be collected at much lower cost, in much larger quantities and without burden on the user. As one of the most effective implicit feedback information, click-through data has been used either as absolute relevance judgments or relative relevance judgments.

To the best of our knowledge, implicit feedback using click through data has not been applied to Web image retrieval systems in both textual space and visual space. Comparing with text retrieval, image retrieval has the following two characteristics. First, since the thumbnail of an image reflects more information than the title and snippet of a Web page, click-through information of image retrieval tends to be less noisy than that of text retrieval.

Second, unlike textual document, the content of an image can be taken in at a glance. As a result, the user will possibly click more results in image retrieval than in page retrieval. Both characteristics imply that click-through data could be more helpful for image retrieval. Thus there has been study on generating semantic features based on user's click-through data for image search results clustering.

Although all existing commercial Web image retrieval systems solely depend on textual information, Web images could be characterized by textual and visual features. Note that making effective use of textual features can conduce image retrieval by high-level concepts more efficient, and leverage mature techniques from text retrieval. However, just as the proverb "a picture is worth one thousand words", the textual representation of an image is always insufficient compared to the visual content of the image itself. Therefore, visual features are required for finer granularity of image description. Considering textual and visual feature, RF in textual space could guarantee relevance and RF in visual space could meet the need for finer granularity.

\section{Features And Metric}

\subsection{Structural Similarity Index Metric:}

The structural similarity (SSIM) metric aims to measure quality by capturing the similarity of images. A product of three aspects of similarity is measured: luminance, contrast, and structure. The luminance comparison function $\mathrm{L}(\mathrm{x}, \mathrm{y})$ for reference image $\mathrm{x}$ and test image $\mathrm{y}$ is defined as

$$
l(x, y)=\frac{2 \mu_{x} \mu_{y}+c_{1}}{\left(\mu_{x}^{2}+\mu_{y}{ }^{2}+\mathrm{c} 1\right)}
$$

where $\mu_{x}$ and $\mu_{y}$ are the mean values of $\mathrm{x}$ and $\mathrm{y}$, respectively, and $\mathrm{C} 1$ is a stabilizing constant.

The contrast comparison function $\mathrm{C}(\mathrm{x}, \mathrm{y})$ is defined similarly as

$$
C(x, y)=\frac{2 \sigma_{x} \sigma_{y}+C_{z}}{\left(\sigma_{x}^{2}+\sigma_{y}^{2}+c 2\right)}
$$

where $\sigma_{x}$ and $\sigma_{y}$ are the standard deviation of $\mathrm{x}$ and $\mathrm{y}$, respectively, and $\mathrm{C} 2$ is a stabilizing constant.

The structure comparison functions $\mathrm{S}(\mathrm{x}, \mathrm{y})$ is defined as 


$$
S(x, y)=\frac{2 \sigma_{x y}+C_{3}}{\sigma_{x} \sigma_{y}+C_{g}}
$$

Where $\sigma_{x y}$ is the correlation between x and y and C3 is also a constant that provides stability.

The SSIM index is obtained by combining the three comparison functions

$$
\operatorname{SsIM}(\mathrm{x}, \mathrm{y})=(\mathrm{l}(\mathrm{x}, \mathrm{y}))^{\alpha} \cdot(\mathrm{c}(\mathrm{x}, \mathrm{y}))^{\beta} \cdot(\mathrm{s}(\mathrm{x}, \mathrm{y}))^{\gamma} \text {. }
$$

the parameters are set as $\alpha=\beta=\gamma=1$ and $\mathrm{C} 3=\mathrm{C} 2 / 2$

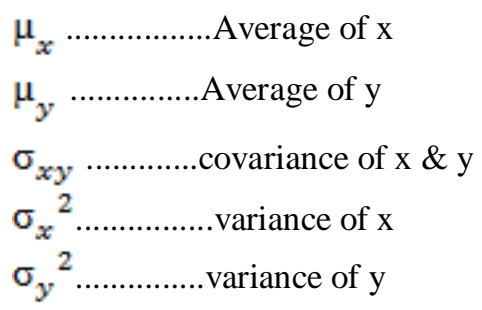

$\mathrm{C} 1=\left(\mathrm{k}_{1} \mathrm{~L}\right)^{2}$

$\mathrm{C} 2=\left(\mathrm{k}_{2} \mathrm{~L}\right)^{2}$

L................Dynamic range of pixel values

$\mathrm{C} 1, \mathrm{c} 2 \ldots \ldots . .$. Two variables to stabilize the division with weak denominator

$\mathrm{K} 1=0.01 ; \mathrm{K} 2=0.03$ by default

Local SSIM statistics are estimated using a symmetric Gaussian weighting function. The mean SSIM index pools the spatial SSIM values to evaluate the overall image quality.

\section{Software Used}

Matlab 10 basic is the software used for the coding of the proposed work.

\section{RESULTS:}

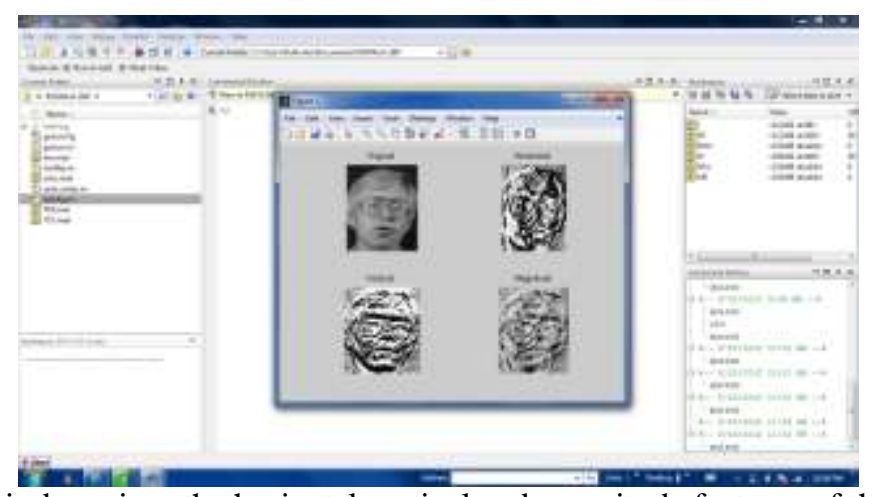

The above window gives the horizotal,vertical and magnitude features of the test image.

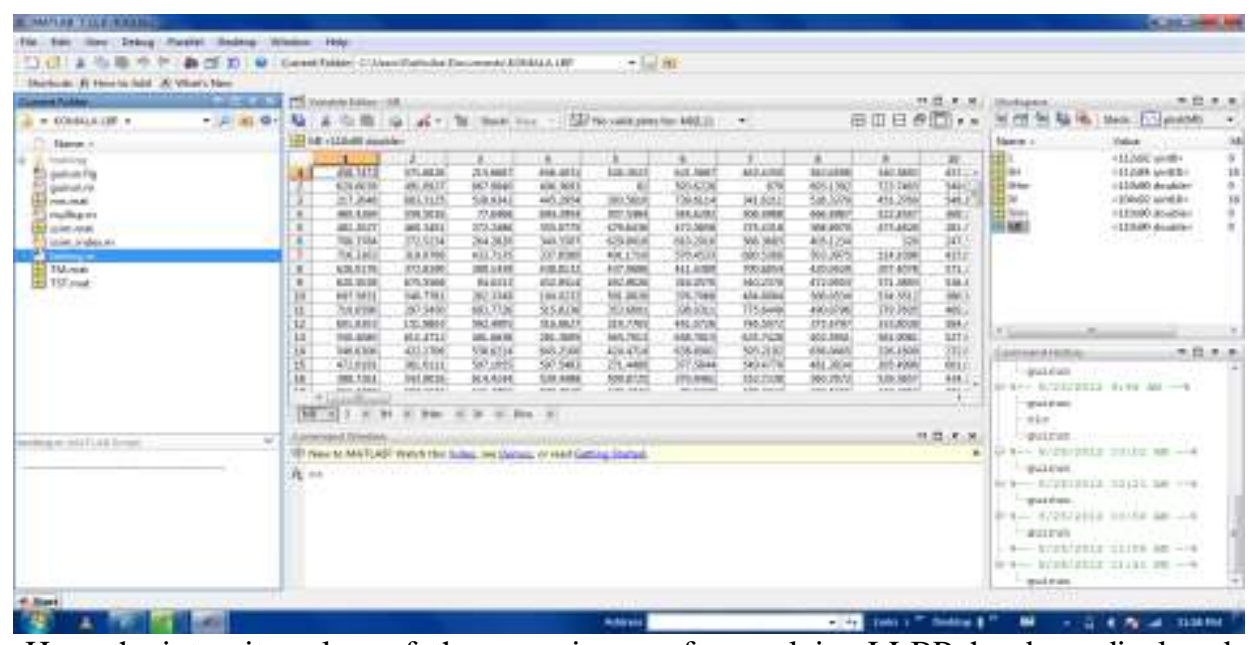

Here, the intensity values of the query image after applying LLBP has been displayed 


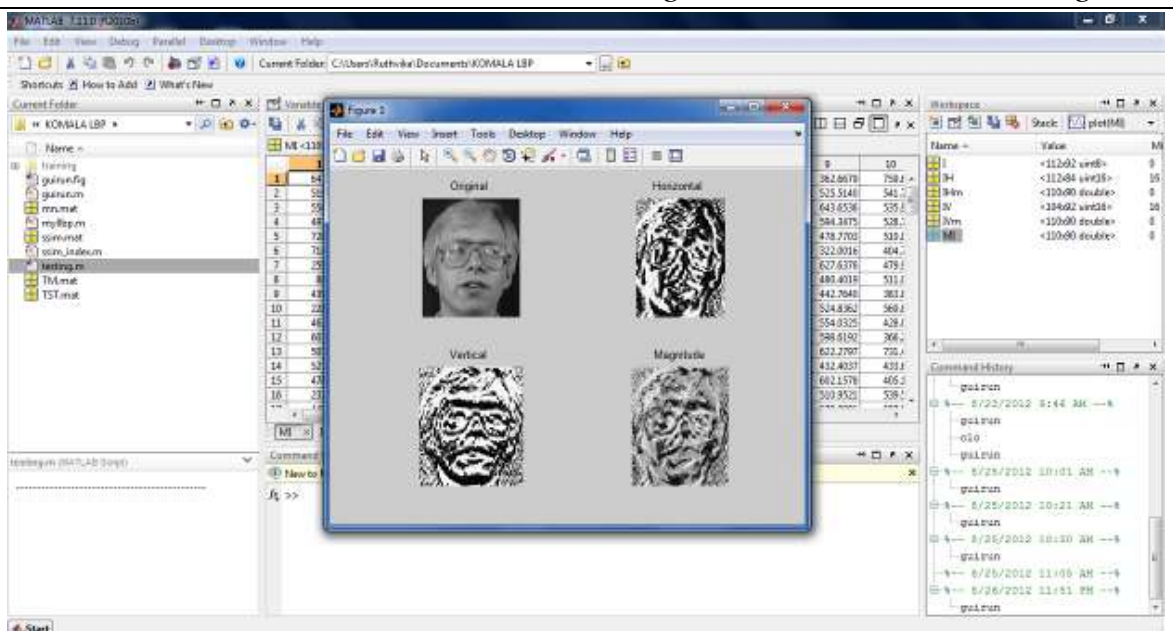

The above window gives the information of the guery image after feedback. Horizontal,vertical and magnitude features were displayed here.

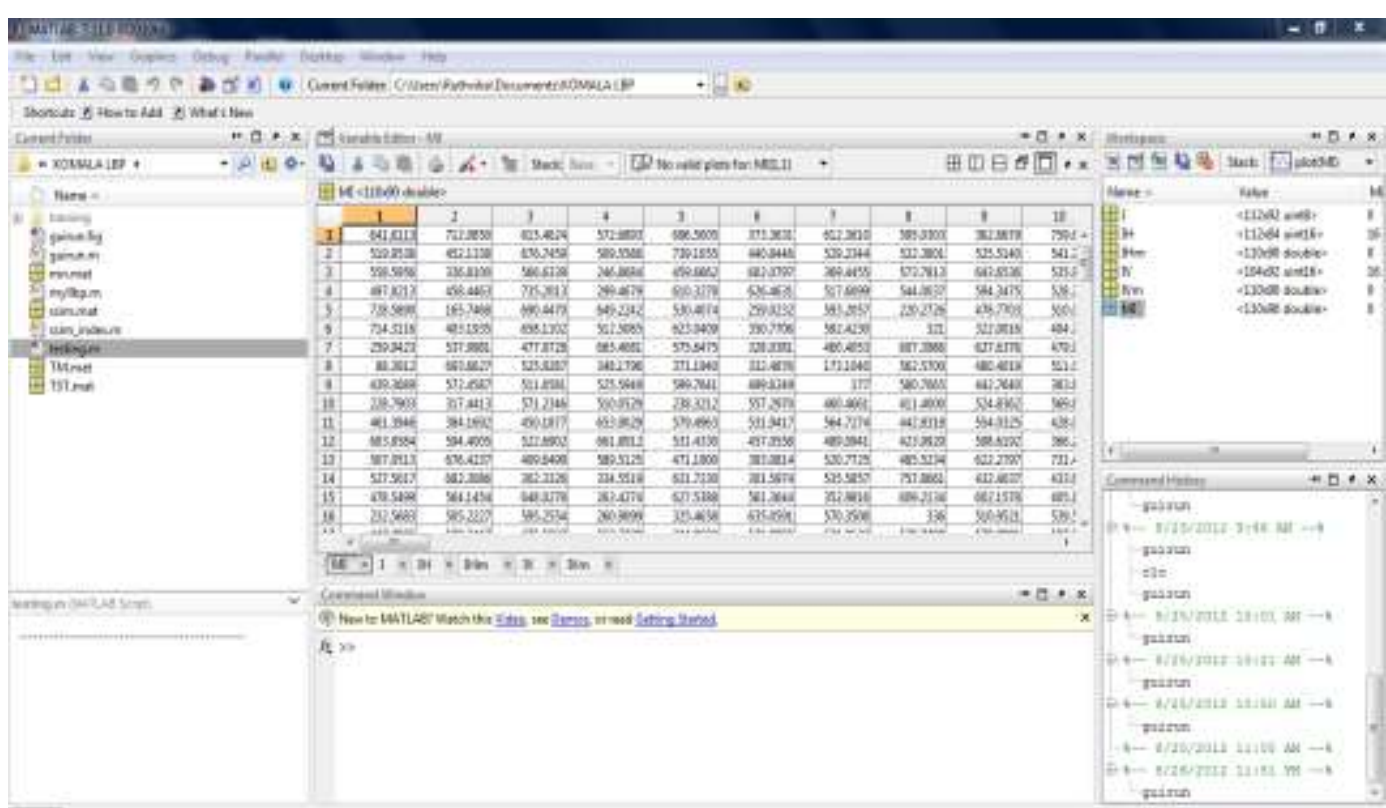

A

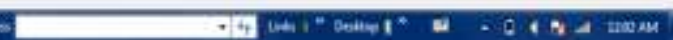

The above window gives the intensity values of the query iamge after feedback. The inteensity values after feedback are high compared to the values before feedback.Hence the recognition rate incresses.

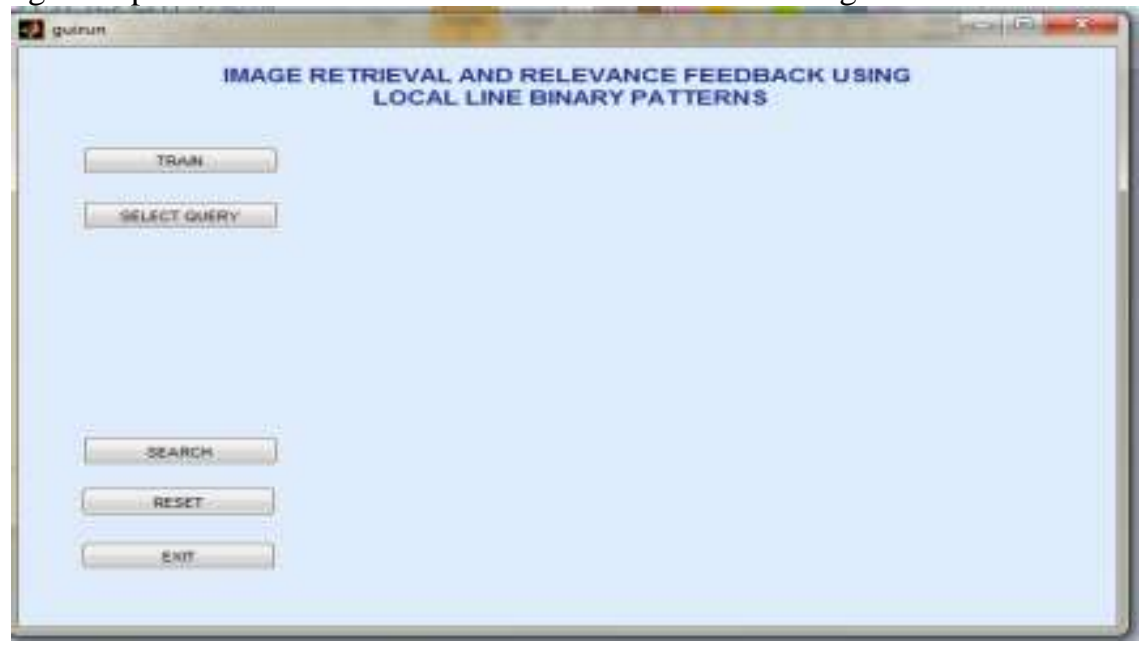

This window appears as and when we type' guirun' in the commandwindow. All the buttons i.e Train,Select Query,Search,Reset,Exit,Index number were created using GUI. 


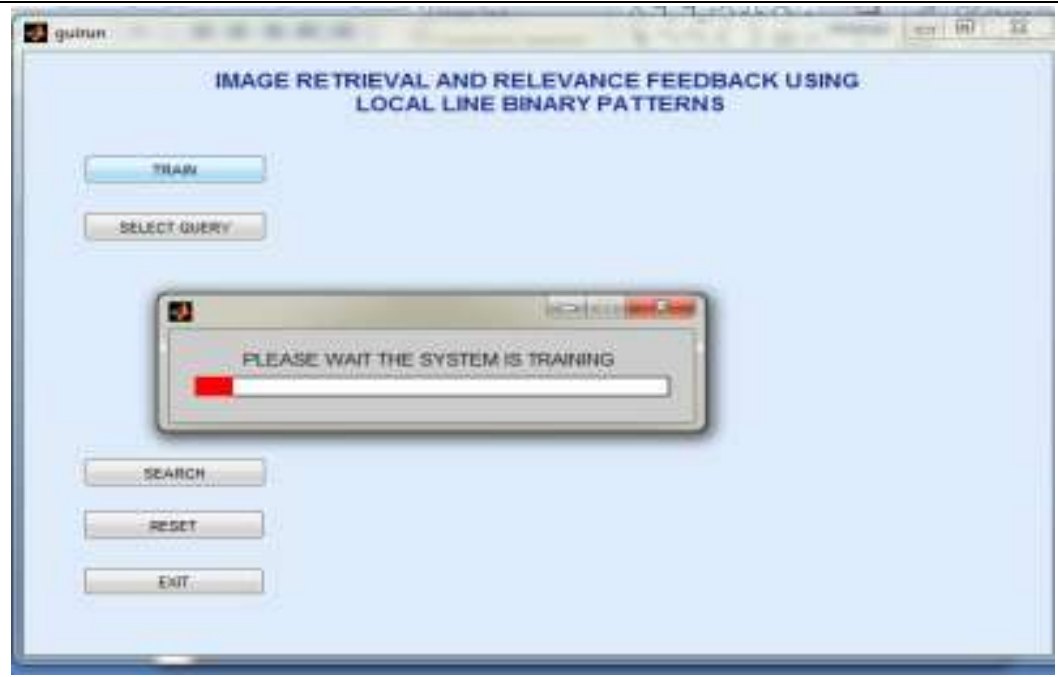

By clicking on the button 'Train'this window appears when the system is training.It means that what all the images available in the database(called as training set or training images) are normalized and then LLBP is applied ,so that texture content has been calculated for the training images.

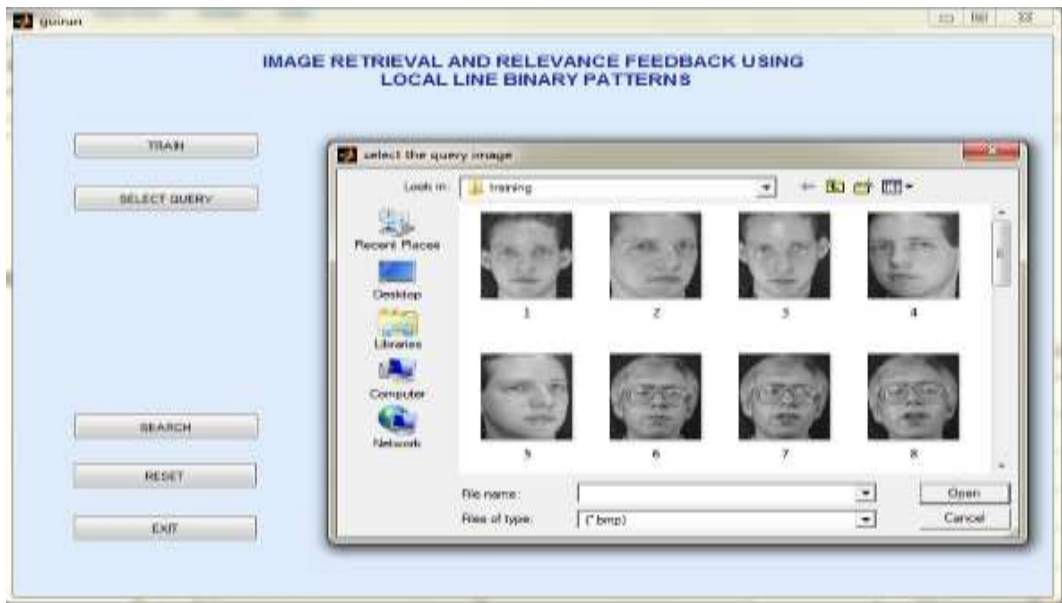

After all the database images were trained,query is selected among them. This is to be done by clicking on a select Query button which is created dy GUI.

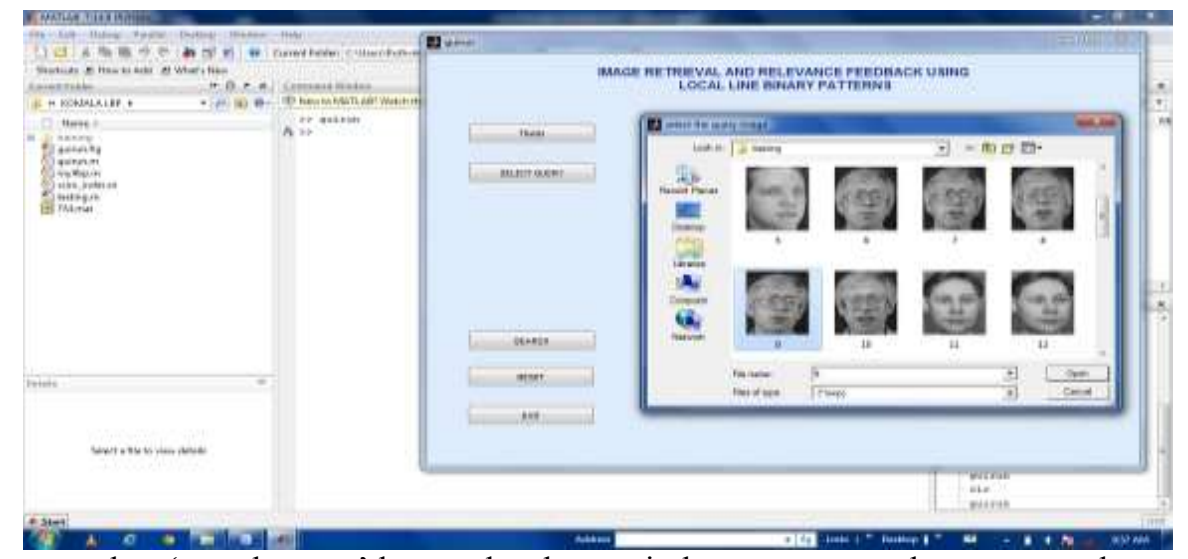

As and when we select 'search query' button,the above window appears on the screen.so that any one image is being selected as a query image.

By observing the above window,the user has choosen 9 as the query image.so it has been selected as the query image. 


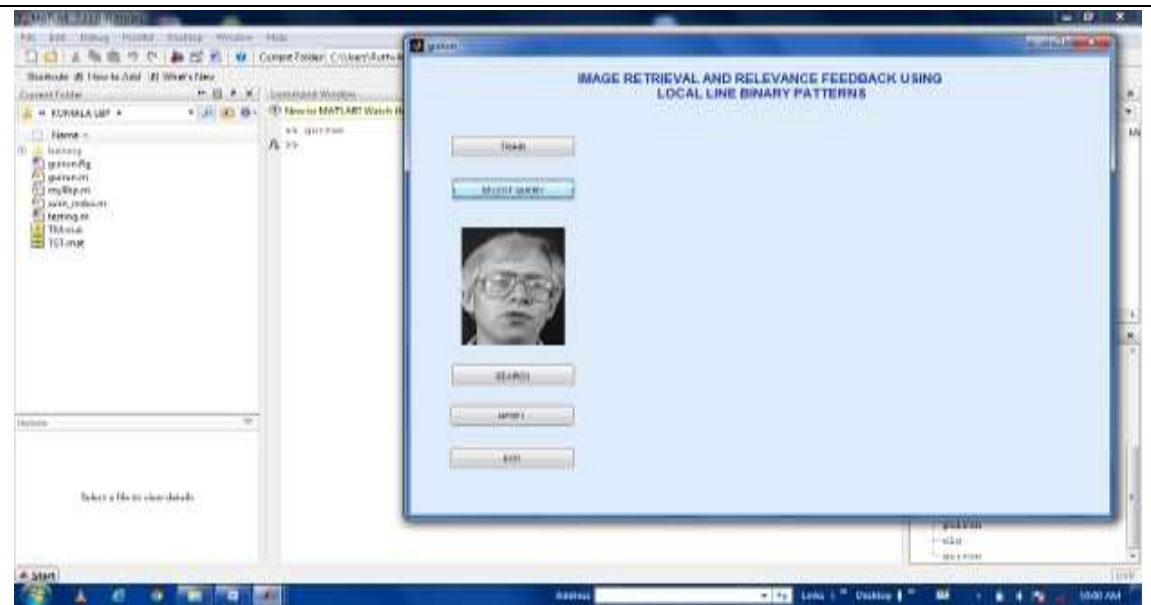

A query has been selected to search in the databasei.e. in the trained images.After selecting the query image,LLBP operator has been applied to obtain the texture content,in order to compare with the training images.prior to LLBP,normalization has also been done to the query image.

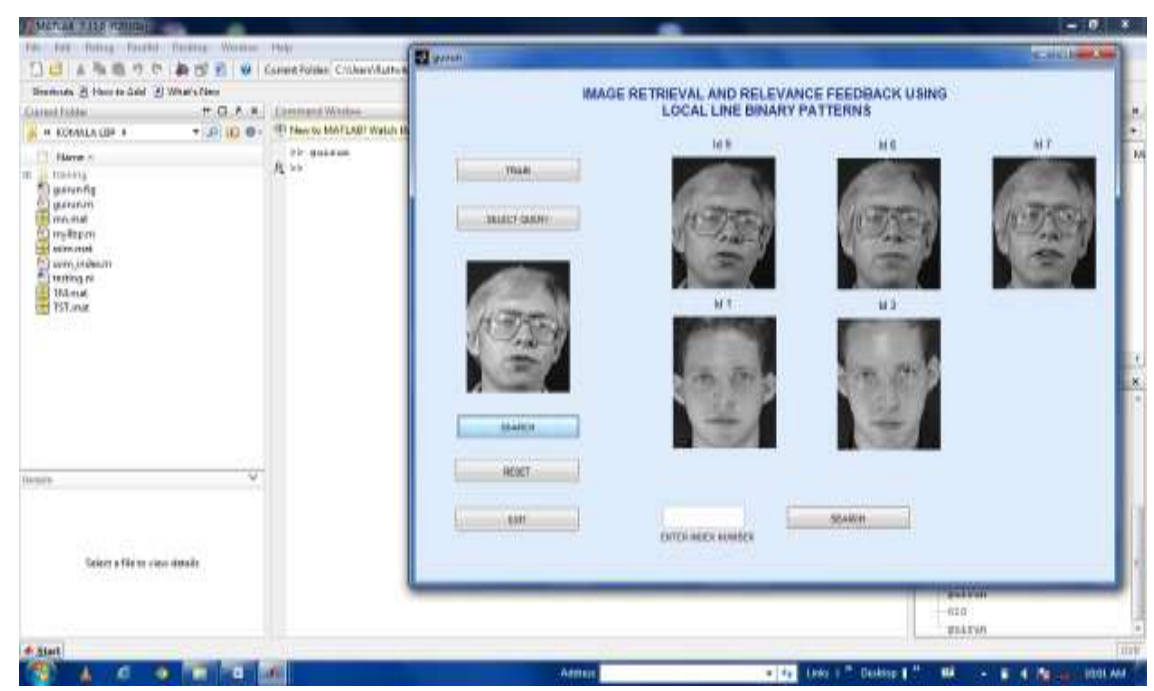

After selecting query, the user click 'search' button,then the most relevant 5 images were displayed from the database corresponding to the query.

By observing the above window,only 3 images are relevant to the query and the remaining 2 images are irrelevant.

To increase the classification rate,feddback mechanism is imlemented.

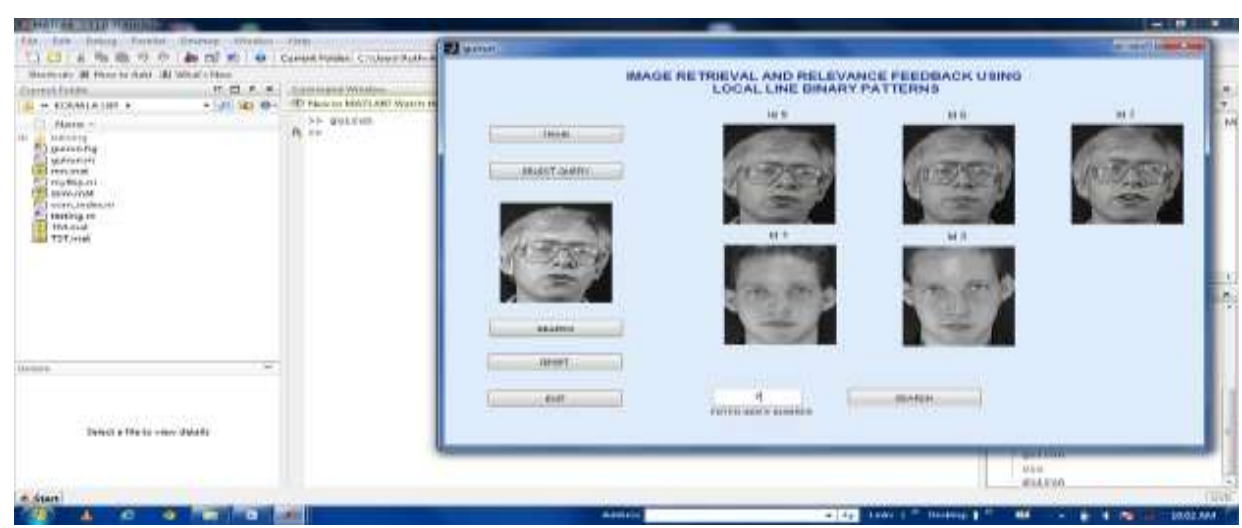

Here,the' index number' button has been created by GUI to enter the id of the image from the 5 most retrieved images in order to give the feedback to get the best results.

Hence the query image has been changed as ' 7 ', i.e the index number has been choosen by the user to get the best results.

After entering index number as ' 7 ', press search. 


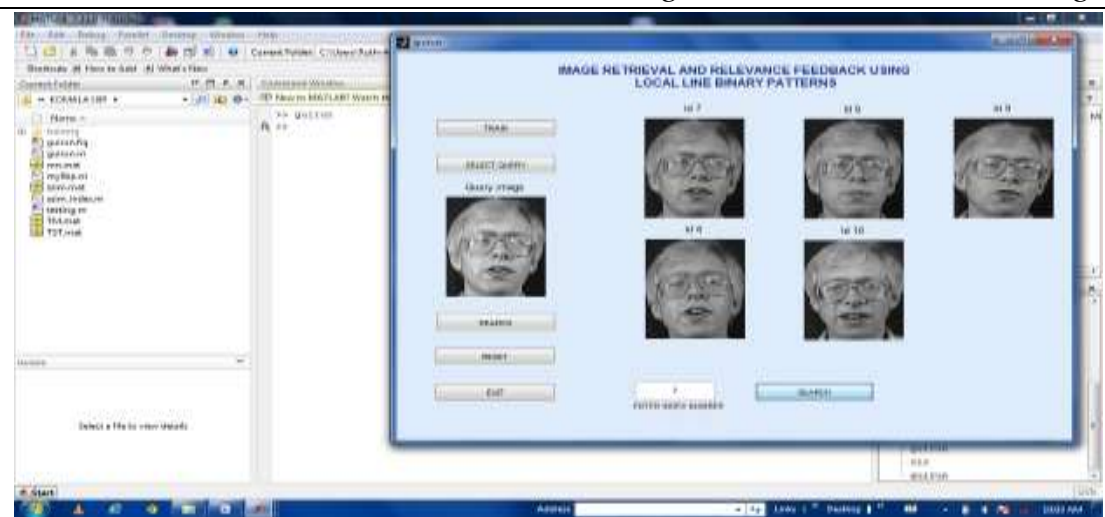

As and when the feedback has been given to the system by entering the index number in the alloted box and press'search' button,again the search process for the query image' 7 ' begins.

The results obtained with query'7' are best compared with query'9'.i.e.all the 5 most relevant images displayed belongs to the query image' 7 '.It means that by applying the feedback,results can be improved.

This is an iterative process, feedback is given iteratively till the user satisfies with the result.

\section{Conclusion}

A novel face recognition method for face recognition has been proposed by using Local Line Binary Pattern( LLBP) which is motivated from Line Binary Pattern(LBP).

5 Most relevant images were displayed by using Content Based Image Retrieval(CBIR) system. To increase the classification rate feedback has been applied to the system. Until the user satisfied with the result, feedback is repeated.Hence this is an iterative process.

Feedback mechanism has been implemented successfully if the user is not satisfied with the results

Limitation: $100 \%$ recognition rate is not possible .

\section{Applications:}

Security

Government Events Criminal

Immigration/Customs

Casino

Toy

Vehicle

\author{
Computer and physical access control \\ Terrorists screening; Surveillance \\ Illegal immigrant detection; Passport/ ID Card authentication \\ Filtering suspicious gamblers /VIPs \\ Intelligent robotic \\ Safety alert system based on eyelid movement
}

\section{Futurescope}

A new method has been proposed for face recognition.Local Line Binary Pattern(LLBP) is applied to recognize a specified face. If the face is not recognized correctly, then more features can be extracted like face colour and moment invariant. Moment Invariants are sensitive features and are used in recognition applications. The face is recognized again using Decision Tree. Applying two stage recognition process increases the recognition accuracy by $2 \%$.

\section{References}

[1] J. Rocchio, Relevance Feedback in Information Retrieval. Upper Saddle River, NJ: Prentice-Hall, 1971.

[2] Ojala, M. Pietik"ainen, and D. Harwood. A comparative study of texture measures with classification based on featured distributions. Pattern Recognition, 29(1):51-59, 1996.

[3] P. N. Belhumeur, J. P. Hespanha, and D. J. Kriegman. Eigenfaces vs. fisherfaces: Recognition using class specific linear projection. IEEE Trans. Pattern Analysis and Machine Intelligence, 20:71-86, 1997.

[4] A [7] G. Guo, S. Z. Li, and K. Chan. Face recognition by supporz vector machines. In Proc. Intl Conf. Automatic Face and Gesture Recognition, pages 196-201, 2000.

[5] T. Ahonen, A. Hadid, Face recognition with local binary pattern,2004.

[6] T. Chen, W. Yin, X. Zhou, D. Comaniciu, and T. Huang. Illumination normalization for face recognition and uneven background correction using total variation based image models. In Proc. IEEE Intl. Conf. Computer Vision and Pattern Recognition, 2005.

[7] T. Chen,W. Yin, X. S. Zhou, D. Comaniciu, and T. S. Huang. Total variation models for variable lighting face recognition. IEEE Trans. Pattern Analysis and Machine Intelligence, 28:1519-1524, 2006.

[8] Q. Tao and R. N. J. Veldhuis. Illumination normalization based on simplified local binary patterns for a face verification system. In Biometrics Symposium 2007 at The Biometrics Consortium Conference, Baltimore, Maryland, pages 1-7, USA, September 2007. IEEE Computational Intelligence Society. [19] Google Image Search, [Online]. Available: http://images.google.com

[9] Yahoo Image Search, [Online]. Available: http://images.search.yahoo. com/

[10] Altavisa Image Search, [Online]. Available: http://www.altavista.com/image/

[11] Picsearch Image Search, [Online]. Available: http://www.picsearch.com 